Original Research Paper

\title{
Restricted Boltzmann Machines for Fundus Image Reconstruction and Classification of Hypertension Retinopathy
}

\author{
${ }^{1,2}$ Bambang Krismono Triwijoyo, ${ }^{3}$ Boy Subirosa Sabarguna, ${ }^{1}$ Widodo Budiharto and ${ }^{1}$ Edi Abdurachman \\ ${ }^{1}$ Doctor in Computer Science, Binus Graduate Program University of Bina Nusantara, Jakarta, Indonesia \\ ${ }^{2}$ Department of Computer Science, University of Bumigora, Mataram, Indonesia \\ ${ }^{3}$ Department of Community Medicine, University of Indonesia, Jakarta, Indonesia
}

\section{Article history}

Received: 31-12-2020

Revised: $18-02-2021$

Accepted: 26-02-2021

Corresponding Author: Bambang Krismono Triwijoyo Department of Computer Science, University of

Bumigora, Mataram, Indonesia Email: bkrismono@ universitasbumigora.ac.id

\begin{abstract}
Conventionally classification of hypertensive retinopathy through analysis of fundus images by experts, but this method the results are highly dependent on the accuracy of observations and expert experience. In this study, we propose a fundus image reconstruction and Hypertensive retinopathy classification model using Restricted Boltzmann Machines (RBM), as well as the Messidor database that has been labeled as a dataset. The experimental results show that the performance of the model produces an accuracy level of $99.05 \%$ where the model can generalize image input into one of the nine classes of the severity of hypertension retinopathy.
\end{abstract}

Keywords: Restricted Boltzmann Machines, Fundus Image, Reconstruction, Classification, Hypertensive Retinopathy

\section{Introduction}

Medical image classification is a challenging research topic, one of which is the retinal image classification which is an important factor in the screening process for eye diseases, including Hypertension Retinopathy [HR] with physical signs of changes in the retinal microvascular as a response to high blood pressure in patients (Wong and Mitchell, 2004). The physical symptoms of retinopathy are narrowing of the retinal vessels, retinal bleeding and cotton white spots.

The conventional method used by ophthalmologists is to evaluate the fundus or retinal images of the eye, to determine the evolutionary phase of hypertensive retinopathy, but this method has a weakness of the traditional method has a weakness in the accuracy and consistency of observations, because it only relies on the eye doctor's vision, especially in the case of early stages of symptoms of hypertensive retinopathy will be difficult to do manually identification (Khitran et al., 2014). Based on these reasons, early diagnosis of hypertensive retinopathy through automatic analysis of retinal images is needed as an aid to the ophthalmologist in screening with accurate results for the prevention and treatment of hypertensive retinopathy.

This study aims to develop a classification model of hypertension retinopathy through in-depth learning methods, using Restricted Boltzmann Machines and analyze the performance of hypertension retinopathy classification models with retinal image input data from the MESSIDOR database.

Restricted Boltzmann Machine (RBM) is a rule of learning using the Boltzmann Machine method (Hinton, 2012). RBM is a probabilistic generative model that can automatically extract data input features using an unsupervised learning algorithm (Hinton, 2002; Smolensky, 1986). RBM uses a recurrent network architecture. Technically, RBM is a stochastic neural network (a neural network which means it has neuron units in the form of binary activations that depend on interconnected neurons, whereas stochastic means activation which has probabilistic elements) which consists of two binary units namely visible layer is stated to be observed and the hidden layer is feature detectors and unit bias. Furthermore, each visible unit is connected to all hidden units represented by an array of weights, so that each hidden unit is also connected to all visible units and bias units are connected to all visible units represents the number of hidden neurons. RBM is controlled by a series of weights and biases in all layers.

In general, the purpose of the RBM algorithm is to rebuild the input as accurately as possible. Then the input is changed based on weight and bias and then used 
to convert the input into an output. In the next stage, the output will be input in the next iteration. At this stage, the input layer tries to change the activation as an input reconstruction and then uses this input to compare with the original input (Ranzato et al., 2010).

In the case of computer vision, each visible unit corresponds to a pixel value from the image while the hidden units represent independent specific features of the image. The weights connecting the visible and the hidden units are usually trained using contrastive divergence learning which is an approximation of maximum likelihood learning (Xia et al., 2016). Methods using RBMs have become more popular in recent years and they are successfully applied to image recognition (Yamashita et al., 2014).

\section{Related Work}

Previous research has used the learning algorithm in RBM as a feature extraction method, proposed by (Hinton and Salakhutdinov, 2006). RBM produces a high ability for feature extraction and representation; Empirical research has proven that using features extracted from the RBM algorithm instead of raw data results in significant improvements in different machine learning applications, such as the classification of color images (Larochelle and Bengio, 2008), speech and object recognition (Li et al., 2015). The learning algorithm in $\mathrm{RBM}$ is designed to extract discriminatory features from large and complex data sets by introducing hidden units in an unsupervised way.

Previous studies relating to the classification of hypertensive retinopathy used features of AVR with datasets DRIVE and VICAVR (Khitran et al., 2014). They used a hybrid classifier which is a combination of Naive Bayes and SVM with accuracy for the DRIVE dataset is $98 \%$ and for VICAVR dataset is $96.5 \%$. The Preprocessing steps are still needed to detect AVR properly and eliminate noise (Abbasi and Akram, 2014), used features of the ratio of Arterial and Venous diameter (AVR). They used 100 images of hypertensive retinopathy patients and used four methods, Artificial Neural Networks (ANN), Naive Bayes, Decision Tree (DT), Support Support Vector Machine (SVM) with an accuracy of 76, 75, 68 and 81\%, respectively. Agurto et al. (2014) used AVR features and the Tortuosity Index, local dataset and they used Partial Least Squares (PLS) methods with $80 \%$ accuracy. This method needs additional features of AV nicking, vascular branching angles and embolic plaque for vascular changes. Cavallari et al. (2015) used the AVR feature and Tortuosity Index, 16 Images of the retina from the local data set. They used the average fractal dimension (mean-D) method with Accuracy results is $68.8 \%$.

The classification of hypertensive retinopathy using deep learning was conducted by (Triwijoyo et al., 2017). The model and dataset used are Convolutional Neural Network (CNN) and DRIVE dataset, with an accuracy of $98.6 \%$.

While (Akbar et al., 2018) proposed detection of hypertensive retinopathy using edge detection of arterial and venous vessels on retinal images from three datasets of INSPIRE-AVR, VICAVR and AVRDB, with 95, 96.8 and $98.8 \%$, respectively. The detection of hypertensive retinopathy using the Neural Network has also been proposed by (Syahputra et al., 2018; Arsalan et al., 2019). Syahputra et al., Used the Backpropagation Neural Network model and the STARE dataset with 95\% accuracy, while Arsalan et al., Used the Vess-Net model and three datasets DRIVE, CHASE-DB1 and STARE with an accuracy of $96.55,97.16 \%$ respectively and $96.97 \%$. Table 1 shows that our proposed method of classification of hypertensive retinopathy using the RBM model and the Messidor dataset yields a better accuracy of $99.05 \%$.

Table 1: Comparison of other related research results

\begin{tabular}{|c|c|c|c|c|}
\hline Researcher & Feature & Method & Dataset & Accuracy $(\%)$ \\
\hline \multirow[t]{2}{*}{$\overline{\text { Khitran et al. (2014) }}$} & AVR & Combination of Naif & DRIVE & 98.00 \\
\hline & & Bayes and SVM & VICAVR & 96.50 \\
\hline \multirow{4}{*}{ Abbasi and Akram (2014) } & AVR & ANN & Local dataset & 76.00 \\
\hline & & Naïve Bayes & & 75.00 \\
\hline & & Decision tree & & 68.00 \\
\hline & & SVM & & 81.00 \\
\hline Agurto et al. (2014) & AVR, Tortuosity Index & Partial Least Squares & Local dataset & 80.00 \\
\hline Cavallari et al. (2015) & AVR, Tortuosity Index & average fractal dimension & Local dataset & 68.80 \\
\hline Triwijoyo et al. (2017) & AVR & CNN & DRIVE & 98.60 \\
\hline \multirow[t]{3}{*}{ Akbar et al. (2018) } & AVR & 2-D Gabor wavelet & INSPIRE-AVR & 95.00 \\
\hline & & Canny Edge Detection & VICAVR & 96.80 \\
\hline & & & AVRDB & 98.80 \\
\hline Syahputra et al. (2018) & AVR & Back-propagation neural network & STARE & 95.00 \\
\hline \multirow[t]{3}{*}{ Arsalan et al. (2019) } & AVR & Vess-Net & DRIVE & 96.55 \\
\hline & & & CHASE-DB1 & 97.16 \\
\hline & & & STARE & 96.97 \\
\hline Our method & AVR & RBM & Messidor & 99.05 \\
\hline
\end{tabular}




\section{Materials and Methods}

In this section, we will discuss dataset inputs, data balancing, architecture and learning algorithms from classification models using RBM.

\section{Dataset}

We used database Methods to Evaluate Segmentation and Indexing Techniques in the Field of Retinal Ophthalmology (MESSIDOR) as a dataset (Messidor, 2010). Messidor is a research program funded by the French Ministry of Research and Defense within a 2004 TECHNO-VISION program. This database can be used, free of charge, only for research and educational purposes. Messidor database consists of 1200 eye fundus color digital images of the posterior pole, which were acquired by three ophthalmologic departments, using a color video 3CCD camera on a Topcon TRC NW6 non-mydriatic retina graph with a 45 degrees field of view. Figure 1 shows an example of fundus images from the Messidor database.

The images saved in uncompressed TIFF format were captured using 8 bits per color plane at $1440 \times 960$, $2240 \times 1488$, or $2304 \times 1536$ pixels resolution.

\section{Method}

Figure 2 shows the diagram of the proposed method, in general, there are six steps. Starting with four steps of preprocessing the input image from the Messidor database, which consists of the cropping and resizing process, segmentation, measuring ARVs and labeling to determine the class of hypertensive retinopathy to produce a new dataset of hypertensive retinopathy consisting of nine classes. Next is the training process of the RBM model and the last is the testing of the training result model using test data to produce a classification of hypertensive retinopathy.

Preprocessing includes cropping the original image to remove the left and right parts of the background image, focus more on the retina image and reduce complexity. The cropping process changes the original image size from $1440 \times 960$ to $900 \times 900$ pixels, from $2240 \times 1488$ to $1380 \times 1380$ pixels and from $2304 \times 1536$ to $1452 \times 1452$ pixels. After the cropping process, then the three sizes of cropped images are resizing to one dimension of $256 \times 256$ pixels to be used as input to the classification model using the Restricted Boltzmann machines.

The sample data from the dataset divided into training datasets and validation datasets. Each category of the class is taken $60 \%$ as data training and $40 \%$ used as data validation. We used a cross-validation training method, with leave-one-out. This method was adopted from (Cawley and Talbot, 2003). The leave-one-out cross-validation resulted in seven times faster training time as well as a relatively lower error rate than the k-fold cross-validation. We calculated the ratio between Arterial and Venous width (AVR) of 89 retinal image samples by adopting (Hubbard et al., 1999; Bhuiyan et al., 2013) methods, the next is segmenting retinal blood vessels, measuring AVR and labeling retinal images in nine classes based on AVR for training model by modifying the category of HR by (Abbasi and Akram, 2014). Table 1 shows the proposed new categorization of hypertensive retinopathy based on AVR.

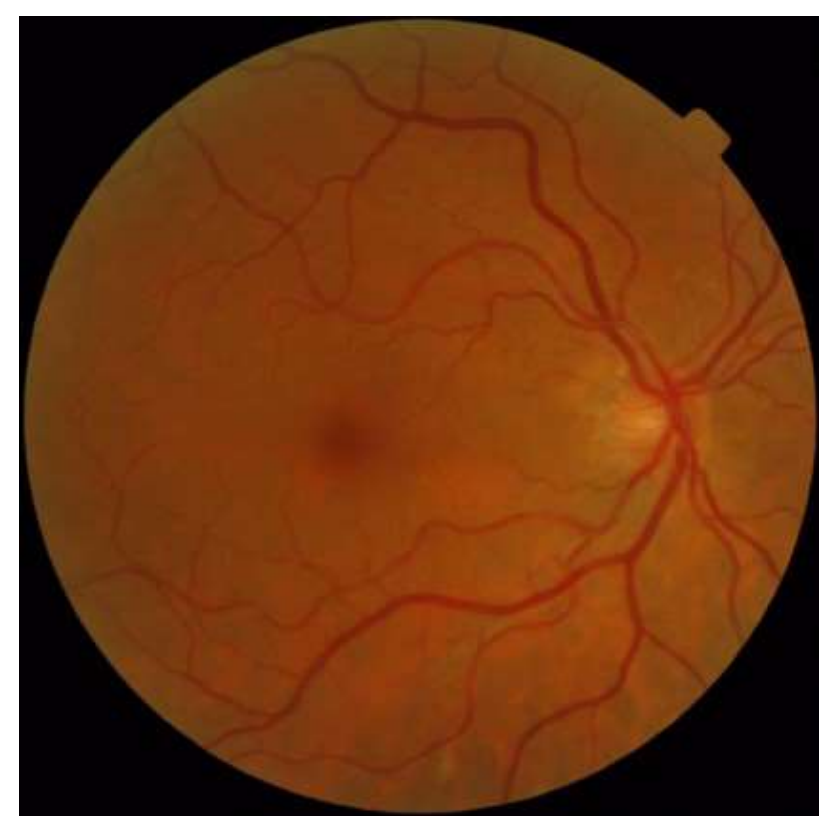

Fig. 1: An example of fundus images from the Messidor database (Messidor, 2010) 


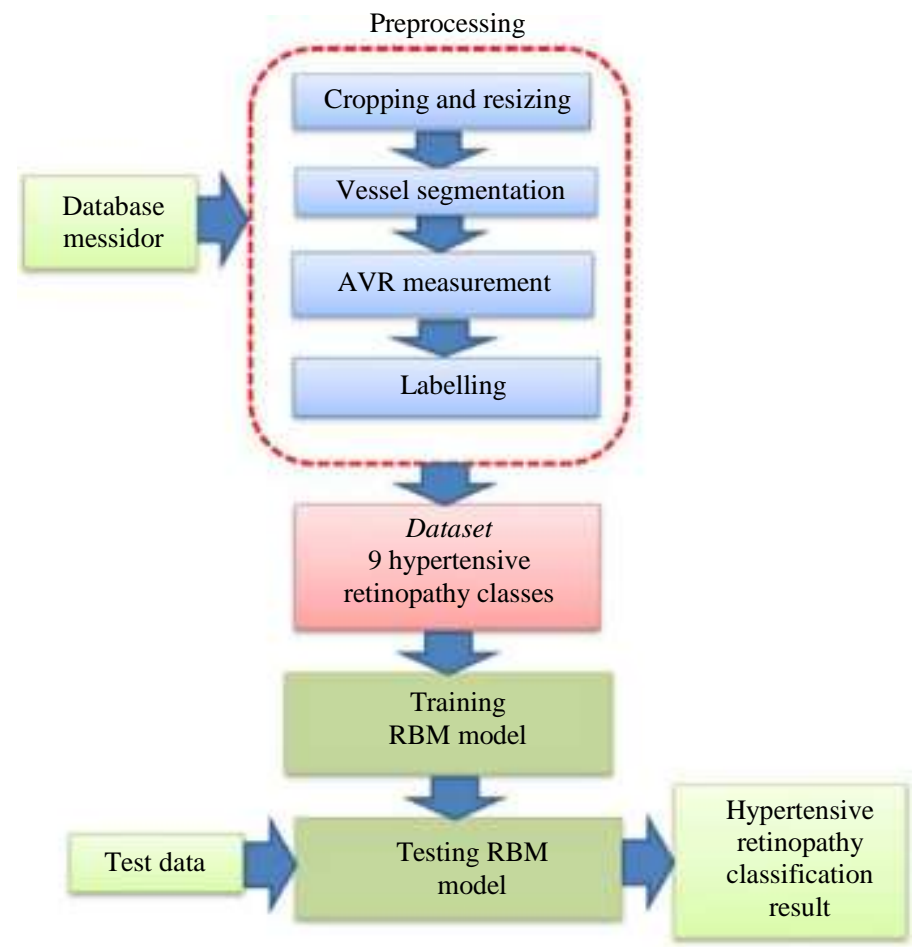

Fig. 2: The Diagram of RBM model for classification of hypertensive retinopathy

Table 1: The category of HR is based on AVR

\begin{tabular}{lr}
\hline Category & AVR \\
\hline Normal & $0.67-0.74$ \\
Borderline 1 & $0.51-0.66$ \\
Stadium 1 & $0.40-0.50$ \\
Borderline 2 & $0.34-0.39$ \\
Stadium 2 & $0.31-0.33$ \\
Borderline 3 & $0.25-0.30$ \\
Stadium 3 & $0.23-0.24$ \\
Borderline 4 & $0.20-0.22$ \\
Stadium 4 & $0-0.19$ \\
\hline
\end{tabular}

Table 2: The results of labeling and balancing data

\begin{tabular}{llrrrr}
\hline Category & Class labels & Number of images & Used & Class labels & Number of images \\
\hline Normal & 0 & 23 & 23 & 110 & 133 \\
Borderline 1 & 1 & 6 & 6 & 127 & 133 \\
Stadium 1 & 2 & 5 & 5 & 128 & 133 \\
Borderline 2 & 3 & 13 & 13 & 120 & 133 \\
Stadium 2 & 4 & 11 & 11 & 122 & 133 \\
Borderline 3 & 5 & 18 & 18 & 115 & 133 \\
Stadium 3 & 6 & 4 & 4 & 130 & 134 \\
Borderline 4 & 7 & 4 & 4 & 130 & 134 \\
Stadium 4 & 8 & 5 & 5 & 129 & 134 \\
\hline
\end{tabular}

Table 2 shows the results of the data labeling process, where the number of retinal images per class is not balanced so that the duplication and augmentation methods are used to add data for classes with less than 133 for class 0 to 5 labels and less than 134 for class 6 to 8 labels. Whereas for classes whose data exceeds 133 for class 0 to 6 labels and more than 134 for class 6 to 8 labels, the amount of data is reduced so that eventually balanced data is obtained and ready for the model training process.

In this study, we used RBM for the classification of hypertension retinopathy based on retinal images. Figure 3 shows an illustration of the architecture of the RBM model for image classification: 


$$
\begin{aligned}
& v \in\{0,1\}^{D} \\
& h \in\{0,1\}^{P}
\end{aligned}
$$

where $v$ is the visible layer, $h$ is the hidden layer, $D$ is the number of visible units and $P$ is the number of hidden units, as well as training datasets in vectors $N$ :

$$
\{v\}_{n=1}^{N}
$$

The RBM model input in the form of a retinal color image, each value of the intensity of the image pixel is read and converted into a value between 0 to 1 , then becomes the input for visible nodes, so the number of visible nodes corresponds to the number of pixels of the input image. Then the first iteration process is adjusting the connection weights between each visible node and each hidden node until we get the output of hidden nodes which then updates the value of the visible node. The process is repeated for the next iteration and until the last epoch.

The training model consists of setting model parameters and model architecture experiment scenarios. While testing is the stage of testing the model that has been carried out in the training phase. In this testing phase, the data test set from the MESSIDOR database was used, where the 30 samples of the data test set were not used in the model training process. The dataset consists of nine classes of hypertensive retinopathy that have been categorized and labeled. The number of epochs is 20, the batch size is 30 and the number of sample images for testing is 30 randomly selected.
The algorithm of Restricted Boltzmann Machines is as follows (Salakhutdinov and Hinton, 2009):

Random initiation of parameters $\theta^{0}$ and $\mathrm{M}$ particles $\left\{v^{0,1}\right.$, $\left.h^{0,1}\right\}, \ldots,\left\{v^{0, M}, h^{0, M}\right\}$

For $t=0$ to $T$ (number of iterations)

For each training data $v^{n}, n=1$ to $N$

Initiate $\mu$ by random and update mean-field until convergent, where:

$$
\begin{aligned}
& \mu_{j} \leftarrow \sigma\left(\sum_{i} W_{i j v_{i}}+\sum_{m / j} J_{m j \mu_{m}}\right) \\
& \text { Let } \mu^{n}=\mu
\end{aligned}
$$

\section{EndFor}

For each particle $m=1$ to $M$

Get the new state $\left(v^{t+1, m}, h^{t+1, m}\right)$ by executing $\mathrm{k}$ stages, initialized in the previous sample $\left(v^{t, m}, h^{t, m}\right)$

$$
\begin{aligned}
& p\left(h_{j}=1 \mid v, h_{-j}\right)=\sigma\left(\sum_{i=1}^{D} W_{i j v_{i}}+\sum_{m=1 \backslash j}^{P} J_{j m} h_{j}\right) \\
& p\left(v_{i}=1 \mid h, v_{-j}\right)=\sigma\left(\sum_{i=1}^{P} W_{i j h_{i}}+\sum_{k=1 \backslash j}^{D} L_{i k} v_{j}\right)
\end{aligned}
$$

EndFor

Update Weight:

$$
W^{t+1}=W^{t}+\alpha_{t}\left(\frac{1}{N} \sum_{n=1}^{N} v^{n}\left(\mu^{n}\right)^{T}-\frac{1}{M} \sum_{m=1}^{M} v^{t+1, m}\left(h^{t+1, m}\right)^{T}\right)
$$

Decrement Learning rate $\alpha \_t$

\section{EndFor}

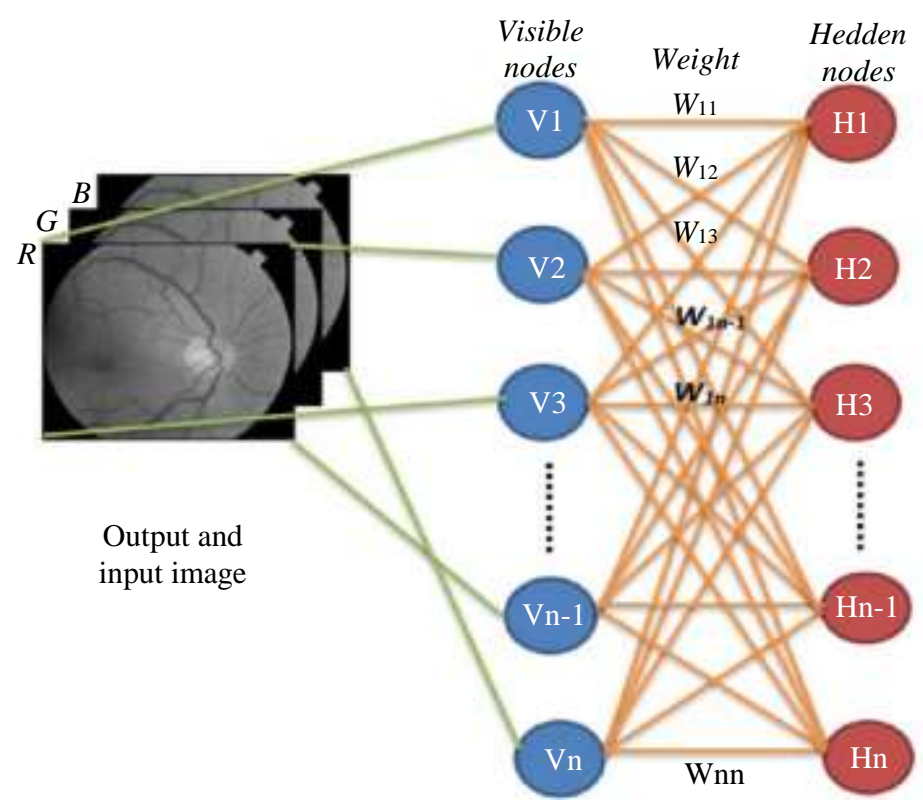

Fig. 3: The RBM model architecture for image classification 
This research was implemented four RBM models with different numbers of visible units each 2352, 12288, 49152 and 196608 units. The learning rate of the four models is 0.05 and the hidden number of the four models is the same as 1500 units. This research also was implemented four types of RBM models with a different number of hidden layers, each of 500, 1000, 1500 and 2000 units. The final part of this section will discuss the results of RBM model experiments with four kinds of learning rate values, each of them are 0.5 , $0.05,0.005$ and 0.0005 .

\section{Results}

The experiments were carried out using specifications hardware and software environment specifications on laptops with Intel Core i7-7500U processor specifications, 12 GB RAM, GPU: NVIDIA GeForce GTX 960, Windows 10 operating system. Python 3.6 Programming Language with a Jupyter notebook.

\section{Experimental Results Using a Different Image Size}

Table 3 shows the results of the training process experiments of four types of RBM models. The number of visible nodes in each RBM model is according to the input image size $28 \times 28 \times 3,64 \times 64 \times 3,128 \times 128 \times 3$ and $256 \times 256 \times 3$. The four RBM models use the same number of hidden layer nodes as 1500 units and a learning rate of 0.05 .

The training performance of the four RBM models is very good, with an accuracy level of both training and validation above $98 \%$. The difference in accuracy from the four RBM models is not too significant or smaller than $0.19 \%$. From these empirical facts, it can be concluded that the size of the input image does not significantly affect the accuracy of the RBM model training results.

As for the training time, there is a significant correlation between the size of the input image and the training time, where the greater the size of the input image, the greater the number of visible nodes of the RBM model, so that it has implications for the longer training time. The accuracy of the results of the testing model shows that the more the number of visible nodes, the less the accuracy of the testing model.

Figure 4 shows a graph of the training results of the four RBM models with varying input image sizes. the blue line is the training error level from epoch 0 to epoch 19 or as many as 20 epochs. While the green line is the validation error level from epoch 0 to epoch 19 . From the four graphs, it appears that at the beginning of epoch 0 to 3 the validation error rate is relatively lower than the error training level, this shows that there is overfitting, but after the third epoch, shows that the error rate training and validation have the same trend until the 20th epoch.

The smaller the size of the input image or the smaller the number of visible nodes, the faster the rate of error reduction and error validation in the RBM model. Finally, the convergence of the error training level and the validation error level of the four types of RBM models occur after the fifth epoch. Based on the three facts above, then for the trial scenario, the next RBM model will use the second model, namely the RBM model with an input size of $64 \times 64 \times 3$ pixels, each pixel of the input image will be read by one visible node, so the total number of visible nodes is 12288 nodes.

\section{Experimental Results Using a Different Number of the Hidden Nodes}

In this experiment, a comparative analysis of four RBM models with the different numbers of hidden nodes was performed, each of which is 500, 1000, 1500 and 2000 nodes. The RBM model is trained up to 20 epochs using the Messidor data set with a learning rate of 0.05 . The number of training data sets is 1200 retinal images with dimensions of $64 \times 64 \times 3$ pixels, $40 \%$ of the data or 480 images are used for validation and a sample of 30 images is used for testing the RBM model. Table 4 shows the fact that first the more the number of hidden nodes the less the accuracy of training and the validation accuracy of the RBM model. Second, the more hidden nodes, the longer the training process.

Table 3: The results of training model with different image size

\begin{tabular}{lllll}
\hline $\begin{array}{l}\text { Image size (Number } \\
\text { of Visible Nodes) }\end{array}$ & $\begin{array}{l}\text { Training } \\
\text { accuracy }(\%)\end{array}$ & $\begin{array}{l}\text { Validation } \\
\text { accuracy }\end{array}$ & Training time & $\begin{array}{l}\text { Testing } \\
\text { accuracy }(\%)\end{array}$ \\
\hline $28 \times 28 \times 3(2352)$ & 99.05 & 99.04 & $51.72 \mathrm{sec}$ & 99.10 \\
$64 \times 64 \times 3(12288)$ & 99.01 & 99.01 & 8.45 min & 99.05 \\
$128 \times 128 \times 3(49152)$ & 98.88 & 98.90 & 39.68 min & 98.96 \\
$256 \times 256 \times 3(196608)$ & 98.86 & 98.89 & $1.88 \mathrm{~h}$ & 98.94 \\
\hline
\end{tabular}

Table 4: The results of training model with different number of hidden nodes

\begin{tabular}{lllll}
\hline The number of hidden nodes & Training accuracy $(\%)$ & Validation accuracy $(\%)$ & Training time & Testing accuracy $(\%)$ \\
\hline 500 & 99.06 & 99.07 & $3.69 \mathrm{~min}$ & 99.11 \\
1000 & 99.01 & 99.01 & $4.91 \mathrm{~min}$ & 99.05 \\
1500 & 99.01 & 99.01 & $8.45 \mathrm{~min}$ & 99.05 \\
2000 & 98.99 & 98.99 & $8.07 \mathrm{~min}$ & 99.04 \\
\hline
\end{tabular}


Bambang Krismono Triwijoyo et al. / Journal of Computer Science 2021, 17 (2): 156.166 DOI: $10.3844 /$ jessp.2021.156.166

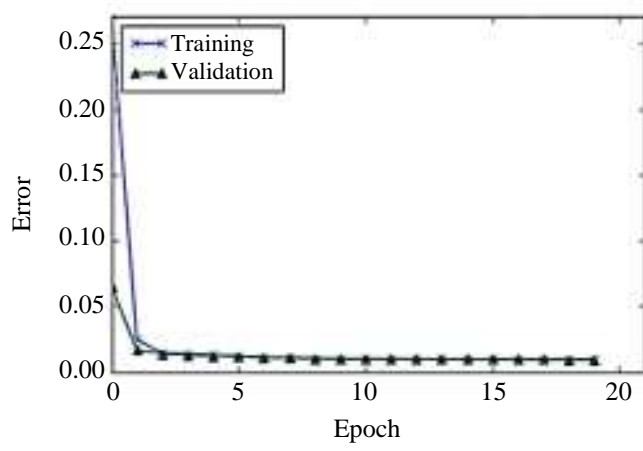

(a)

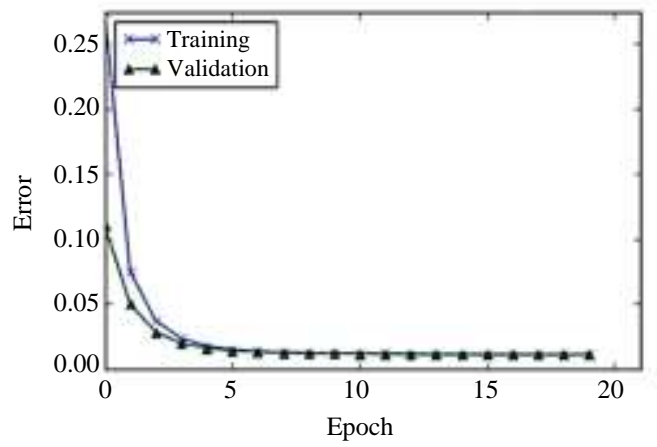

(c)

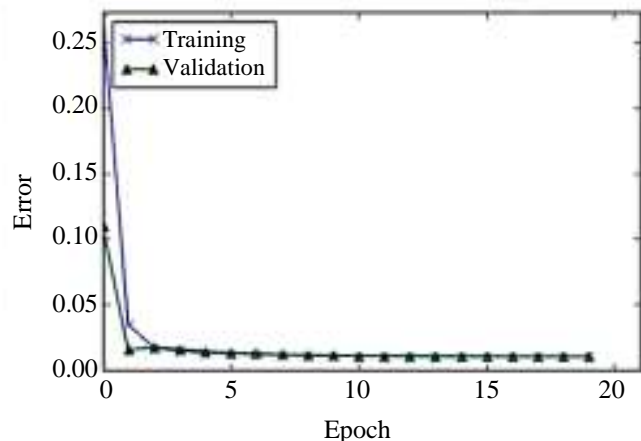

(b)

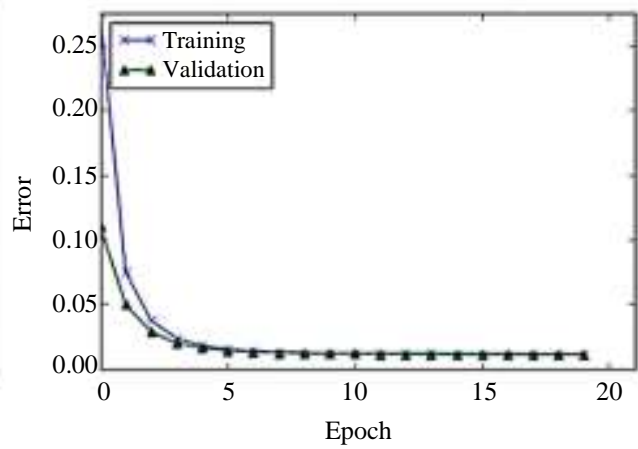

(d)

Fig. 4: Error Training and Validation of the RBM model with The Size of The Input Image (a) $28 \times 28 \times 3$, (b) $64 \times 64 \times 3$, (c) $128 \times 128 \times 3$ and (d) $256 \times 256 \times 3$ pixels

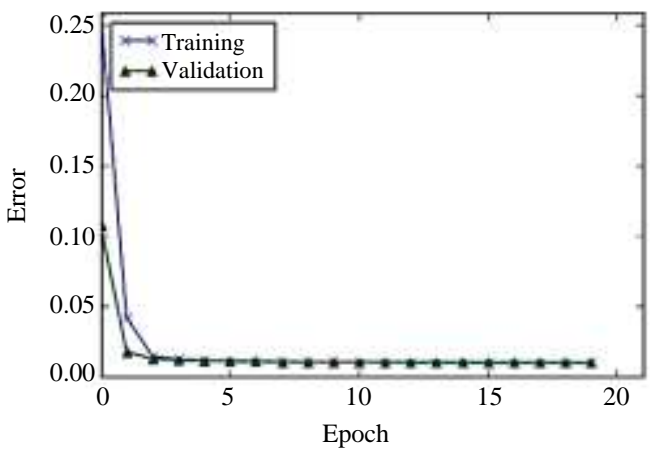

(a)

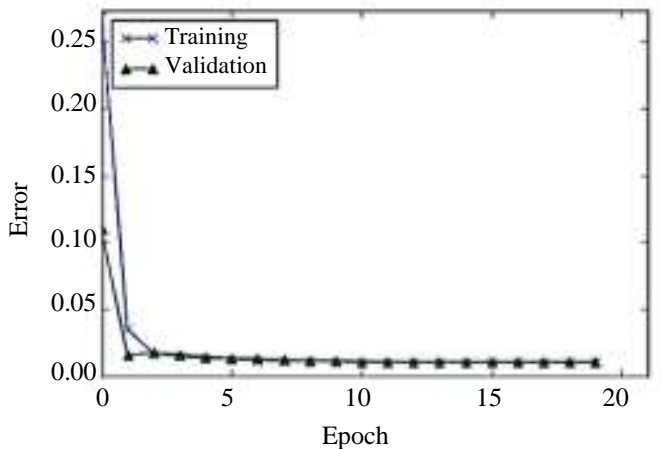

(c)

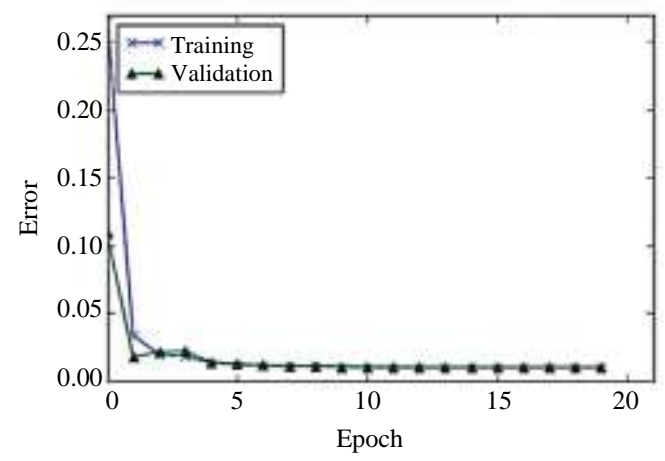

(b)

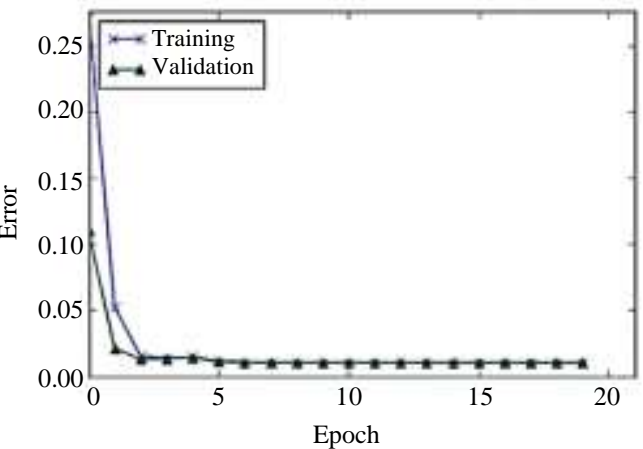

(d)

Fig. 5: Error training and validation of the RBM model with the number of hidden nodes (a) 500, (b) 1000, (c) 1500 and (d) 2000 nodes 
Third, the more number of hidden nodes, the less testing accuracy of the RBM model, the difference is very small or not too significant.

Figure 5 shows a graph of the training results of the four RBM models with varying the number of hidden nodes. the blue line is the training error level from epoch 0 to epoch. While the green line is the validation error level from epoch 0 to epoch 19. From the four graphs, it appears that the performance of the RBM model is almost the same as the results of the model trials using variations in the number of visible nodes, where at the beginning of epoch 0 to 5 the validation error rate is relatively lower than the training error level, this shows that there is overfitting, but after the fifth epoch, showing that error and validation training had the same trend until the 19th epoch

The fewer the number of hidden nodes, the faster the rate of error reduction and error validation in the RBM

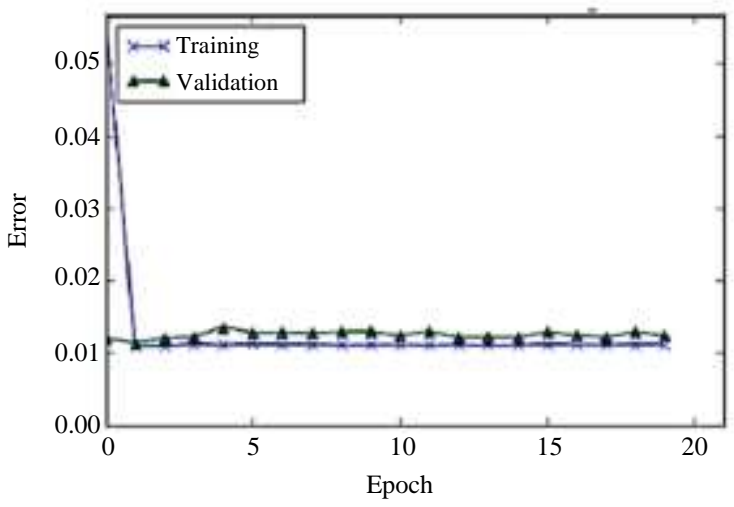

(a)

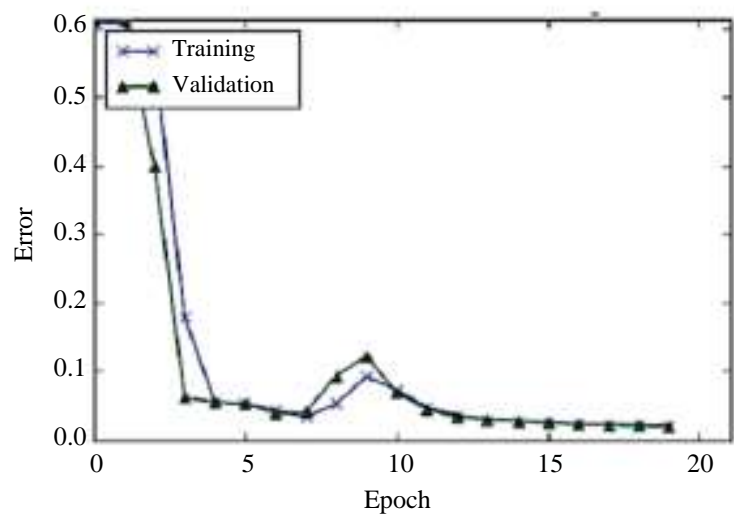

(c) model. Finally, the convergence of the training error training rate and the validation error rate of the four types of RBM models occurred after the tenth epoch.

\section{Experimental Results Using a Different Learning Rate}

This section describes the results of RBM model experiments that have model specifications with the number of visible nodes 12288 and the number of hidden nodes 1500 . Then try using three kinds of learning rate values, each of them is $0.5,0.05,0.005$ and 0.0005 . The RBM model is trained up to 20 epochs using the Messidor data set. The number of training data sets is 1200 retinal color images with dimensions of $64 \times 64$ pixels, $40 \%$ of the data or 480 images are used for validation and a sample of 30 images is used for testing the RBM model.

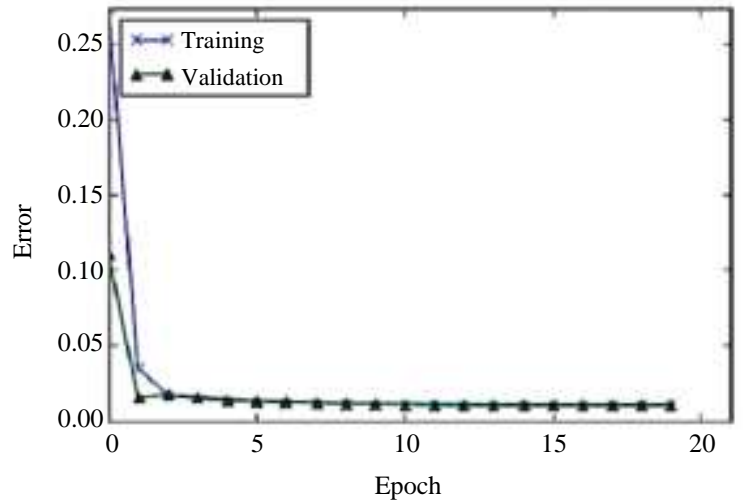

(b)

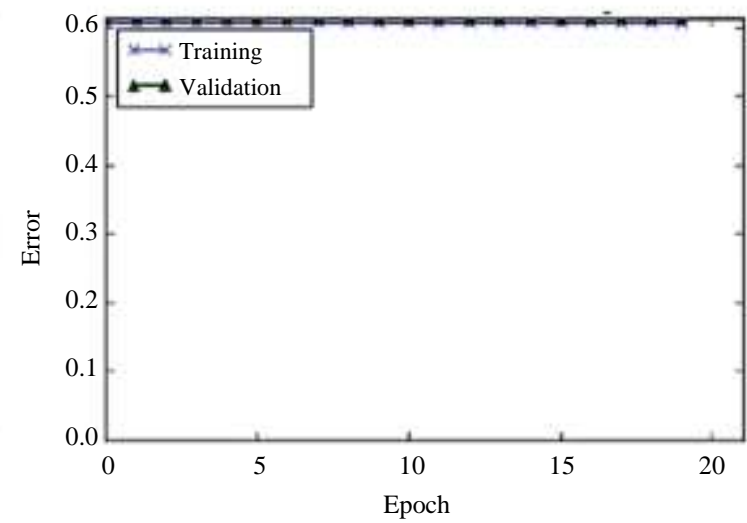

(d)

Fig. 6: Error training and validation of the RBM model with learning rate (a) 0.5 , (b) 0.05 , (c) 0.005 and (d) 0.0005

Table 5: The results of training model with different learning rate

\begin{tabular}{lllll}
\hline Learning rate & Training accuracy $(\%)$ & Validation accuracy $(\%)$ & Training time & Testing accuracy $(\%)$ \\
\hline 0.5 & 98.89 & 98.76 & $6.77 \mathrm{~min}$ & 98.83 \\
0.05 & 99.01 & 99.01 & $8.44 \mathrm{~min}$ & 99.05 \\
0.005 & 98.03 & 98.09 & $6.01 \mathrm{~min}$ & 98.30 \\
0.0005 & 39.19 & 38.59 & $6.12 \mathrm{~min}$ & 38.97 \\
\hline
\end{tabular}


Table 5 shows that first, up to a learning rate of 0.005 the level of training accuracy, validation accuracy and the testing accuracy is relatively stable above $98 \%$, but at a learning rate of 0.0005 , the level of training accuracy, validation and testing drastically drops to the range of $39 \%$. This empirically proves that the learning rate of the ideal RBM model is greater than 0.005 . While the training time for the four types of selective RBM models is around $6 \mathrm{~min}$, except for the RBM model with a learning rate value of 0.05 , where the training time is $8.44 \mathrm{~min}$, despite having the highest level of training accuracy, validation and testing among the four types of RBM models that tested.

Figure 6 shows a graph of the training results of the four RBM models with varying the number of hidden nodes. the blue line is the training error level from epoch 0 to epoch. While the green line is the validation error level from epoch 0 to epoch 19. Based on the four graphs of the experimental results it can be concluded that the RBM model with a learning rate of 0.5 and 0.05 has a relatively similar trend in performance levels of training errors and validation. In the model with a learning rate value of 0.005 , there are fluctuations in the level of training errors and validation in epochs 7 to 11 , although at the end of the 20th epoch the three types of RBM models above have relatively similar levels of training and validation errors below $2 \%$.

From the three experimental scenarios as well as the empirical data of the experimental results, the RBM model with the number of hidden nodes 1500 and learning rate 0.05 is the best performance RBM model. The analysis and discussion of the experimental results of the Retinopathy Hypertension Classification Model using RBM were concluded, first, the model could reconstruct the input image into one of the image classes with a relatively small error rate. Second, the RBM training time is relatively faster than the other model.

The model that we propose from the results of this study is still very open for further development. The usefulness of this result study is: First, a new dataset for classification of hypertensive retinopathy into nine classes, which can be used as a standard dataset for other researchers to test their proposed model. Second, the RBM classification model can be implemented for the classification of retinal images that experience noise, because the RBM model is capable of reconstructing images. Third, the model we propose can be implemented for the classification of other medical images such as images of the prostate, lungs and others. Fourth, the model that we propose can be developed as a tool for ophthalmologists in assisting the diagnosis and early detection of hypertensive retinopathy, based on the patient's retinal image.

\section{Discussion}

The contributions of this study are: First, a new dataset of hypertensive retinopathy which consists of nine classes according to the degree of severity based on AVR as an indicator of class categorization and labeling, from retinal images taken from the Messidor database. Second, the experimental results of the Retinopathy Hypertension Classification Model using RBM prove that the model can reconstruct the input image into one of the image classes with a relatively small error rate.

Comparison of the results of the classification of hypertensive retinopathy between the previous research method and the method we proposed, as presented in Table 1, the average previous research used the AVR feature extraction process through segmentation where the method depends on the feature extraction algorithm, while the method we use is the deep learning approach. with the RBM method, where input in the form of retinal images and feature extraction is carried out by the model in the image classification process and our method is proven to produce better accuracy.

The limitations of this study are: First, the output is in the form of reconstructed images, not class labels so that it is still necessary to add layers for classification such as Softmax or Support Vector Machines (SVM) so that the output is in the form of classes. label as in the case of the classification of hypertensive retinopathy. Second, the accuracy of the model is strongly influenced by the amount of labeled training data, where the greater the amount of labeled training data, the higher the model accuracy results. In our study we only used a sample of 89 labeled training data, which were then developed using the duplication and augmentation technique to become 1200 images, taking into account the balancing of each training data class, as presented in Table 2.

Our future work is to develop a hypertensive retinopathy classification model architecture by combining the RBM model with $\mathrm{CNN}$ and other machine learning, in addition to increasing the number of datasets for training and involving experts in image labeling, so that it is expected to improve model performance. The next stage is implementing the model by building an interface on a mobile application to support telemedicine.

\section{Conclusion}

This research is to develop a classification model of hypertension retinopathy using RBM, the experimental results show that the model's performance is very good at reconstructing images with an accuracy rate of $99.05 \%$, meaning that the model has a good ability to generalize image input into one of nine output classes. But the output model is still an image so it needs to be combined with layers like SoftMax, to get the class label output. Our next research plan is to develop a Classification Model for Hypertension Retinopathy by combining Restricted Boltzmann machines with Convolutional Neural Networks to get better classification results in the form of class labels. 


\section{Acknowledgment}

The author would like to thank the availability of the MESSIDOR dataset used in this study. This research study was supported by Bumigora University, Indonesia.

\section{Author's Contributions}

Bambang Krismono Triwijoyo: Preparing, formatting and writing a draft manuscript. He designs a research plan, collects the necessary data and develops the technical implementation of the proposed research.

Boy Subirosa Sabarguna: Provide input related to the medical aspects of this research. He guides and active in scientific discussions and reviews script writing.

Widodo Budiharto: Providing input on the method used in the research. He guides and active in scientific discussions and reviews script writing.

Edi Abdurachman: Coordinating all research work. He guides and critically reviews manuscripts to ensure significant intellectual content.

\section{Ethics}

No ethical issues may arise after the publication of this manuscript. All fundus images presented in this study are publicly available without information about the patient's identity

\section{References}

Abbasi, U. G., \& Akram, M. U., (2014). Classification of blood vessels as arteries and veins for diagnosis of hypertensive retinopathy. In 2014 10th International Computer Engineering Conference (ICENCO), Dec. 29-30, IEEE Xplore Press. Egypt, (pp. 5-9). DOI: 10.1109/ICENCO.2014.7050423.

Agurto, C., Joshi, V., Nemeth, S., Soliz, P., \& Barriga, S. (2014). Detection of hypertensive retinopathy using vessel measurements and textural features. In 2014 36th Annual International Conference of the IEEE Engineering in Medicine and Biology Society, Aug. 26-30, IEEE Xplore Press. Chicago, (pp. 5406-5409). https://doi.org/10.1109/ICENCO.2014.7050423

Akbar, S., Akram, M. U., Sharif, M., Tariq, A., \& Khan, S. A. (2018). Decision support system for detection of hypertensive retinopathy using arteriovenous ratio. Artificial intelligence in medicine, 90, 15-24. https://doi.org/10.1016/j.artmed.2018.06.004.

Arsalan, M., Owais, M., Mahmood, T., Cho, S. W., \& Park, K. R. (2019). Aiding the diagnosis of diabetic and hypertensive retinopathy using artificial intelligence-based semantic segmentation. Journal of Clinical Medicine, 8(9), 1446-1474. https://doi.org/10.3390/jcm8091446
Bhuiyan, A., Kawasaki, R., Lamoureux, E., Ramamohanarao, K., \& Wong, T. Y., (2013). Retinal artery-vein caliber grading using color fundus imaging. Computer Methods and Programs in Biomedicine, 111(1), 104-114. https://doi.org/10.1016/j.cmpb.2013.02.004

Cavallari, M., Stamile, C., Umeton, R., Calimeri, F., \& Orzi, F., (2015). Novel method for automated analysis of retinal images: results in subjects with hypertensive retinopathy and CADASIL. BioMed Research International. https://doi.org/10.1155/2015/752957

Cawley, G. C., \& Talbot, N. L., (2003). Efficient leaveone-out cross-validation of kernel fisher discriminant classifiers. Pattern Recognition, 36(11), 2585-2592. https://doi.org/10.1016/S00313203(03)00136-5

Hinton, G. E., \& Salakhutdinov, R. R., (2006). Reducing the dimensionality of data with neural networks. Science, 313(5786), 504-507. https://doi.org/10.1126/science. 1127647

Hinton, G. E., (2002). Training products of experts by minimizing contrastive divergence. Neural computation, 14(8), 1771-1800. https://doi.org/10.1162/089976602760128018

Hinton, G. E., (2012). A practical guide to training restricted Boltzmann machines. In Neural networks: Tricks of the trade (pp. 599-619). Springer, Berlin, Heidelberg. https://doi.org/10.1007/978-3-64235289-8_32

Hubbard, L. D., Brothers, R. J., King, W. N., Clegg, L. X., Klein, R., \& and Atherosclerosis Risk in Communities Study Group (1999). Methods for evaluation of retinal microvascular abnormalities associated with hypertension/sclerosis in the Atherosclerosis Risk in Communities Study. Ophthalmology, 106(12), 2269-2280. https://doi.org/10.1016/S0161-6420(99)90525-0

Khitran, S., Akram, M. U., Usman, A., \& Yasin, U. (2014). Automated system for the detection of hypertensive retinopathy. In 2014 4th International Conference on Image Processing Theory, Tools and Applications (IPTA), Oct. 14-17, IEEE Xplore. Paris. (pp. 1-6). https://doi.org/10.1109/IPTA.2014.7001984

Larochelle, H., \& Bengio, Y. (2008). Classification using discriminative restricted Boltzmann machines. In Proceedings of the 25 th international conference on Machine learning, Jul. 5-9, Finland, (pp. 536543). https://doi.org/10.1145/1390156.1390224

Li, M., Miao, Z., \& Ma, C., (2015). Feature extraction with convolutional restricted Boltzmann machine for audio classification. In 2015 3rd IAPR Asian conference on pattern recognition (ACPR), Nov. 3-6, IEEE Xplore, Malaysia, (pp. 791-795). https://doi.org/10.1109/ACPR.2015.7486611 
Messidor. (2010). Methods to evaluate segmentation and indexing techniques in the field of retinal ophthalmology. https://www.adcis.net/en/thirdparty/messidor/

Ranzato, M.A., Krizhevsky, A., \& Hinton, G. (2010). Factored 3-way restricted Boltzmann machines for modeling natural images. In Proceedings of the thirteenth international conference on artificial intelligence and statistics. May 13-15, Italy (pp. 621-628).

http://proceedings.mlr.press/v9/ranzato10a.html

Salakhutdinov, R., \& Hinton, G. (2009). Deep Boltzmann machines. In Proceedings of the Twelfth International Conference on Artificial Intelligence and Statistics. Apr. 16-18. MLR Press, USA, (pp. 448-455). http://proceedings.mlr.press/v5/salakhutdinov09a

Smolensky, P. (1986). Information Processing in Dynamical Systems: Foundations of Harmony Theory. In: Rumelhart, D. E., \& McClelland, J.L., Eds., Parallel Distributed Processing, Vol. 1, MIT Press, Cambridge, 194-281. ISBN:9780262291408.

Syahputra, M. F., Amalia, C., Rahmat, R. F., Abdullah, D., Napitupulu, D., Setiawan, M. I., \& Andayani, U. (2018). Hypertensive retinopathy identification through retinal fundus image using backpropagation neural network. In Journal of Physics: Conference Series 978(1), 012106. https://doi.org/10.1088/17426596/978/1/012106.
Triwijoyo, B. K., Budiharto, W., \& Abdurachman, E. (2017). The classification of hypertensive retinopathy using convolutional neural network. Procedia Computer Science, 116, 166-173. https://doi.org/10.1016/j.procs.2017.10.066

Wong, T. Y., \& Mitchell, P., (2004). Hypertensive retinopathy. New England Journal of Medicine, 351(22), 2310-2317. https://doi.org/10.1056/NEJMra032865

Xia, J. Y., Li, X., \& Liu, Y. X. (2016). Application of a new restricted Boltzmann machine to radar target recognition. In 2016 Progress in Electromagnetic Research Symposium (PIERS). Aug. 8-11, IEEE Xplore, China, (pp. 2195-2201). IEEE. https://doi.org/10.1109/PIERS.2016.7734910

Yamashita, T., Tanaka, M., Yoshida, E., Yamauchi, Y., \& Fujiyoshii, H. (2014). To be Bernoulli or to be Gaussian, for a restricted Boltzmann machine. In 2014 22nd International Conference on Pattern Recognition. Aug. 24-28, IEEE Xplore, Sweden, (pp. 1520-1525). 